\title{
Nanopore-Based DNA Analysis via Graphene Electrodes
}

\author{
Qing Zhao, ${ }^{1}$ Yang Wang, ${ }^{1}$ Jianjin Dong, ${ }^{1}$ Lina Zhao, ${ }^{2}$ X. F. Rui, ${ }^{3}$ and Dapeng $Y{ }^{2}$ \\ ${ }^{1}$ State Key Laboratory for Mesoscopic Physics, School of Physics, Peking University, Beijing 100871, China \\ ${ }^{2}$ CAS Key Lab for Biomedical Effects of Nanomaterials and Nanosafety, Institute of High Energy Physics, \\ Chinese Academy of Sciences, Beijing 100049, China \\ ${ }^{3}$ Link A Media Devices Corporation, Santa Clara, CA 95051, USA
}

Correspondence should be addressed to Qing Zhao, zhaoqing@pku.edu.cn and Dapeng Yu, yudp@pku.edu.cn

Received 21 September 2011; Accepted 22 February 2012

Academic Editor: Sulin Zhang

Copyright ( 2012 Qing Zhao et al. This is an open access article distributed under the Creative Commons Attribution License, which permits unrestricted use, distribution, and reproduction in any medium, provided the original work is properly cited.

We propose an improvement for nanopore-based DNA analysis via transverse transport using graphene as transverse electrodes. Our simulation results show conspicuous distinction of tunneling current during translocation of different nucleotides through nanopore. Applying the single-atom thickness property of graphene, our findings demonstrate the feasibility of using graphene as transverse electrodes in future rapid and low-cost genome sequencing.

\section{Introduction}

Nanopore-based DNA analysis is a fast rising star as a singlemolecule technique, and most apt to be the next generation of DNA sequencing [1]. With longitudinal electric field, DNA molecule is forced to pass through the pore with nanometer scale, presenting obvious current blockage signal by blocking the ion flux through the pore. Pioneer works have been done with biological pores and channels, such as $\alpha$-hemolysin [2], which provides a predefined, repeatable, and precise pore structure atomically and a series of limitations, like short lifetime and sensitivity to environment conditions, as well. To overcome these disadvantages, solidstate nanopore [3] was introduced with the developing technology of nanofabrication [4], which shows great durability, possibility for geometry controlling, and compatibility with semiconductor industries [5]. Real-time DNA sequencing is still a big challenge nowadays because the method of longitudinal current detection only focused on the distinctive geometry and structure of four kinds of nucleotides, and great challenge remains such that the nanopore is too thick to realize single-base resolution and translocation of single base is too fast for recording (microseconds per base) [6]. An alternative approach in measuring the transverse current to get single-base resolution was put forward by Zwolak and Ventra in 2005 [7]. The basic idea is when the bases are passing one by one through a voltage-biased tunnel gap inside a solid-state nanopore, they will alternately change the tunneling current based on how the localized base states contribute to the tunneling current since different bases have different local electronic densities of states with different spatial extent owing to their different chemical composition [8]. Intensive calculation work had been reported based on transverse current of different electrodenucleotide couplings, spreading from the influence of noise [9] and environment [10] to the modification of electrodes [11]. However, most of them encountered the problem of interference of adjacent nucleotides when they are in the nanopore simultaneously. Since the length of DNA molecule is $0.32 \mathrm{~nm}$ per nucleotide, much smaller than the thickness of most available materials for transverse electrodes, it is hard to distinguish the neighboring nucleotides, even using the electrodes comprised of $3 \times 3$ gold atoms arranged as a (111) surface $[8,12]$.

Graphene is a two-dimensional hexagonal carbon lattice that was recently discovered [13] and has attracted intensive research attention due to its unique mechanical and electric properties [14]. Its single-atom thickness, ability to survive large transmembrane pressures, and intrinsic conducting properties [15] make it particularly attractive in DNA sequencing field since it holds hope for enabling transverse conductance measurements with single-base resolution. 


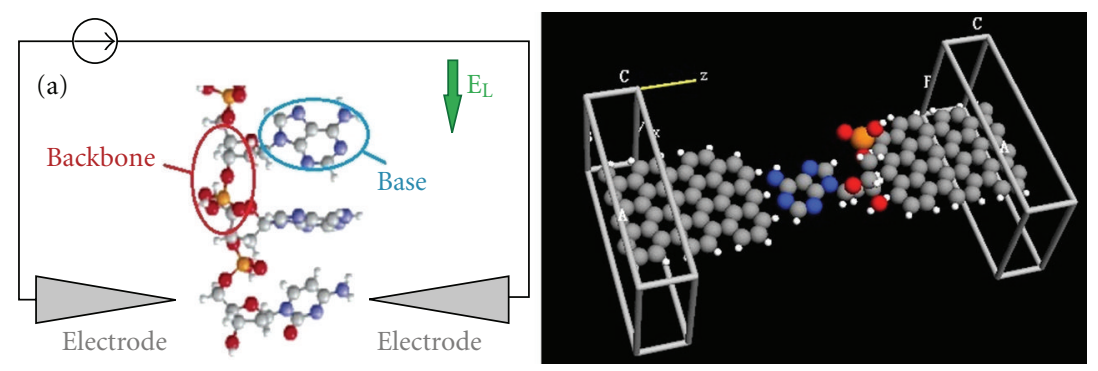

FIGURE 1: Principal diagram of nanopore-based DNA analysis: ssDNA is driven through a nanopore with two graphene electrodes by a longitudinal electric field; the transverse graphene electrodes are applied on defined voltage and used to measure the transverse current. The graphene electrodes are $8.6 \AA$ wide and $12.1 \AA$ apart. The SiN membrane and insulating cover layer are hidden in the graph.

DNA translocation experiment through graphene nanopores has been reported recently by three independent groups [16$18]$, while, in their study, graphene only acts as supporting membrane for nanopores, instead of the transverse electrode to measure tunnelling current. Making graphene as transverse electrode to resolve DNA conductance with single-base resolution experimentally is still very challenging nowadays. A numerical simulation was presented for achieving DNA transverse conductance via graphene nanogap [19], making grapheme-based transverse conductance a promising candidate for robust DNA sequencing. However, nanogap may allow several DNA sequences to pass the gap simultaneously, which may lead to interference problems.

Herein, we propose and theoretically demonstrate a quantum transport simulation using graphene to comprise the transverse electrodes embedded in an SiN nanopore, which can avoid the problem of simultaneous DNA sequence translocation from the nanogap since nanopore has less dimension size compared with nanogap. SiN is used here to act as supporting membrane for graphene. From the simulation results, there exists a perfect exponential relation between the transport current and transverse voltage applied to the electrodes within an appropriate range, and, fortunately, significant distinction between different nucleotides has been demonstrated, implying the feasibility of this method in rapid genome sequencing.

\section{Model and Method}

Figure 1(a) presents the working principle of a nanoporebased DNA analysis: ssDNA is forced to translocate a nanopore by a longitudinal electric field $\left(E_{L}\right)$, and the transverse current is measured via graphene transverse electrodes which are applied on defined voltage. Figure 1(b) shows the cross-section view of our simulation system: single-layer graphene electrodes are embedded in a $6 \mathrm{~nm} \mathrm{SiN}$ layer and covered with a $1 \sim 2 \mathrm{~nm}$ insulating layer, which are hidden in the graph. The graphene electrodes are $7.4 \AA$ wide and $12.1 \AA$ apart, which is also the diameter of the nanopore in the SiN membrane. SiN membrane is used because it is most commonly used in DNA translocation experiment in solid-state nanopore field. The diameter is wide enough for a ssDNA to pass through and narrow enough to hamper two ssDNAs to translocate simultaneously and achieve a measurable tunneling current. Figure 1(b) only shows part of the graphene electrodes and one nucleotide of a DNA sequence, since the rest parts of the nanopore device do not affect the tunneling effect and the thickness of electrodes is much smaller than the scale of the nucleotide molecule.

The simulation process is implemented as follows. First, the position of the nucleotides were set by manual control, in order to get an appropriate coupling of the maximum tunneling current with the electrodes' atoms. The relative positions of four kinds of nucleotides to the graphene electrodes were set up the same, located by the same part, the deoxyribose, of the nucleotides. Then the system of this time section, in which the base is in the flat location of the graphene electrodes, providing the strongest and most characteristic signals of tunneling current in the entire translocation process, was calculated by Atomistix ToolKit (ATK), a quantum transport simulation software, within the extended Hückel method, a semiempirical quantum chemistry method, developed by Hofmann in 1963 considering both pi and sigma orbitals [20]. Using this method, we can get the electronic distribution of the system and deduce the corresponding electric properties, such as the transmission spectra and so on. Finally, transverse currents from the transmission spectra were calculated via nonequilibrium Green's function method [21] and the current curves were contrasted to make our final conclusion. Regarding the issue of strand orientation as it passes through the nanopore, previous calculations showed that the proposed graphene nanopore device is essentially insensitive to strand orientation [22]. Therefore, we did not pay special attention to strand orientation issue during simulation.

Figures 2(a)-2(d) show the transmission spectra of adenosine, thymidine, cytidine, and guanosine under the same bias voltage of $2.4 \mathrm{~V}( \pm 1.2 \mathrm{~V}$ on each graphene transverse electrode), respectively. Because the transmission coefficient of each nucleotide, as well as the tunneling current, is quite different from each other, nearly one or two orders, to hold the maximums of four diagrams the same, we use different scale in each figure. In Figure 2, we could easily pick out the unique and characteristic resonance levels for each kind of nucleotide. We see clearly the characteristic resonance levels for each kind of nucleotide, which results from their unique base types. This could be the foundation of real sequencing in the future. Here, the "windows" of the 


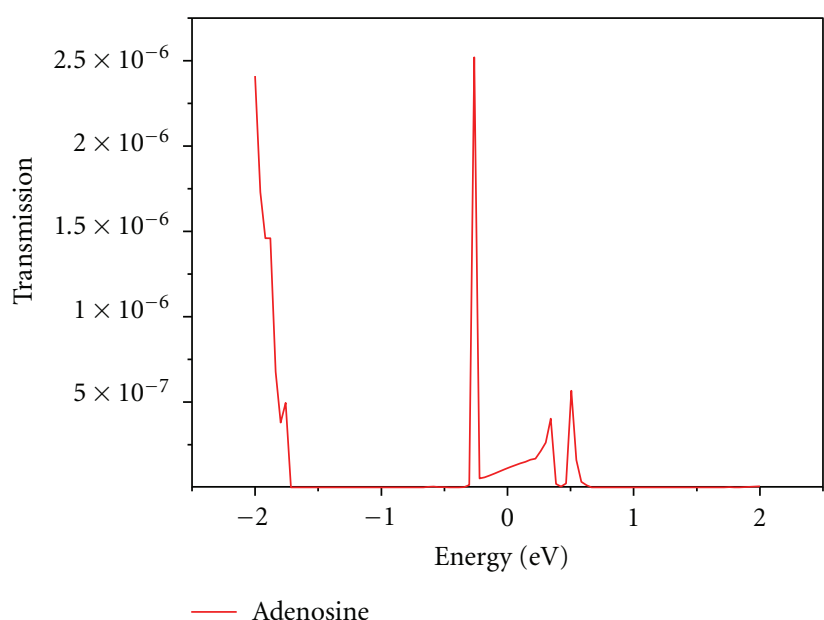

(a)

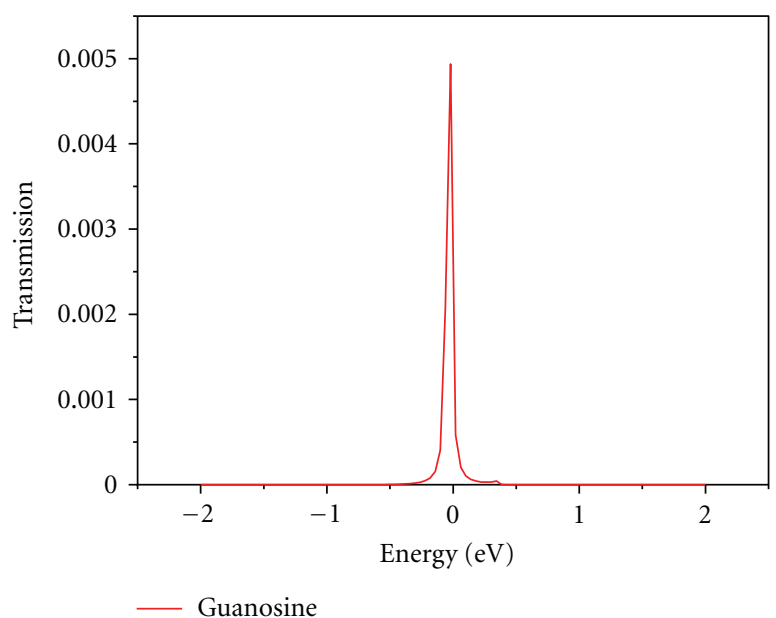

(c)

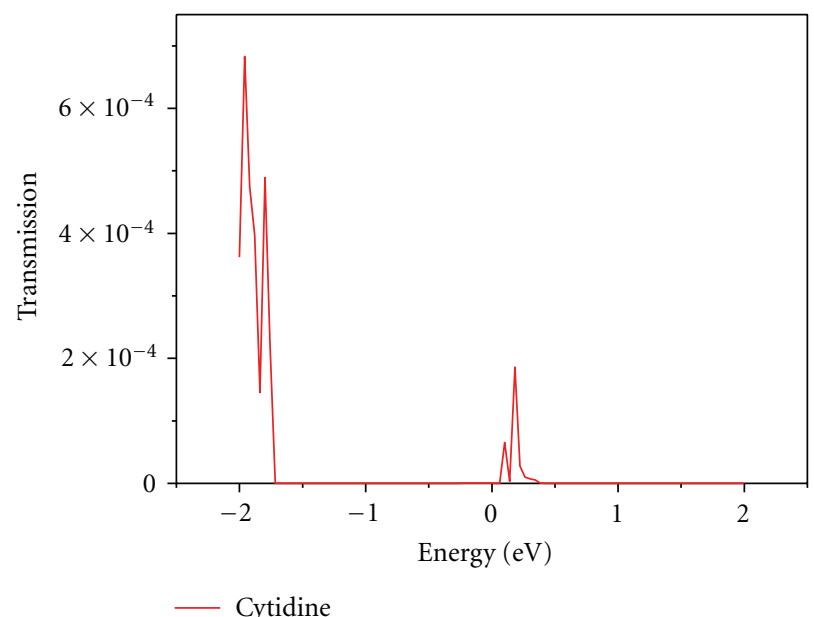

(b)

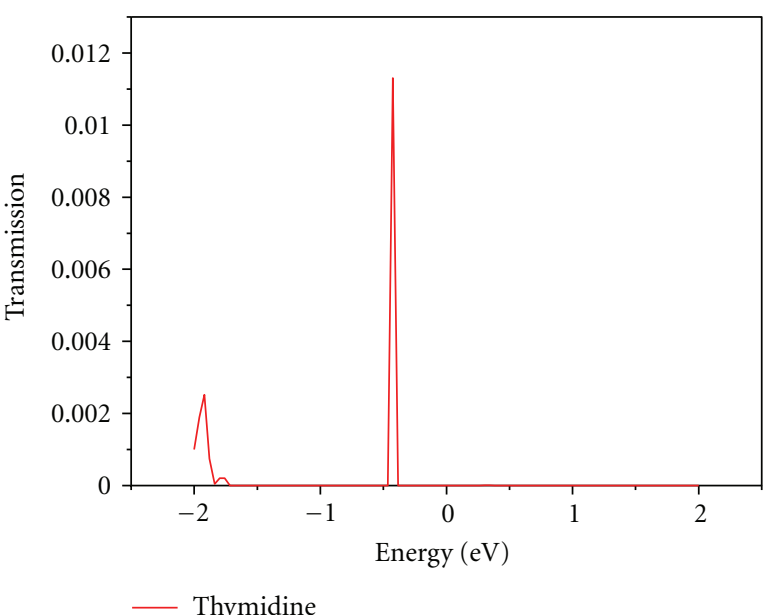

(d)

FIgURE 2: ((a)-(d)) The transmission spectra of adenosine, cytidine, guanosine, and thymidine under the same bias voltage of $2.4 \mathrm{~V}( \pm 1.2 \mathrm{~V}$ on each electrodes, which determine the "window" of the transmission), respectively. Because the transmission coefficient of each nucleotide, as well as the tunneling current, is quite different from each other, nearly one or two orders; to hold the maximums of four diagrams the same, we use different scale in each figure.

spectra are determined by $\varepsilon_{R}$ and $\varepsilon_{L}$, the bias voltages applied on the graphene electrodes.

Consider the well-known formula for the transverse current, as follows [23]:

$$
I_{L R}(V)=\frac{2 e}{h} \int_{-\infty}^{\infty} d \varepsilon T_{L R}(\varepsilon)\left[f\left(\varepsilon-\varepsilon_{L}\right)-f\left(\varepsilon-\varepsilon_{R}\right)\right]
$$

in which, $L, R$ imply the left and right graphene electrode of our system, $V=\varepsilon_{L}-\varepsilon_{R}$ is the bias voltage, $T_{L R}$ is the transmission coefficient, shown in Figure 2, which is calculated from the Hamiltonian matrices of the system, and $f\left(\varepsilon-\varepsilon_{(L / R)}\right)$ is the Fermi distribution function. The equation shows that the transverse electrical current is proportional to the sum of the transmission peaks inside the bias voltage range, which is called the "window." By this method, the tunneling current could be calculated easily with a Python script of ATK, using the extended Hückel method.

\section{Simulation Results and Discussion}

Figure 3(a) is $V$ - $\ln I$ curve of adenosine base pair with bias from $0.0 \mathrm{~V}$ to $3.0 \mathrm{~V}$ with a step of $0.2 \mathrm{~V}$. Here, when voltage is below $1.0 \mathrm{~V}$, the tunneling current is very small, almost hard to detect, as demonstrated in $\ln I$ value. $\ln I$ from -45 to -42 means a null current, which is verified by a simulation carried out on non-load electrode system, where no DNA molecule was used. The current curve of non-load electrodes, shown in Figure 3(a), gave all points below $10^{-19} \mathrm{~A}$. The $V-\ln I$ curve of adenosine clearly shows that, under $1.0 \mathrm{~V}$, the current approximates zero, suggesting the close of the tunneling. The current quickly rises from $1.0 \mathrm{~V}$ to $1.4 \mathrm{~V}$, and, when the voltage is above $1.4 \mathrm{~V}$, the current behaves a perfect exponential relation with the bias voltage. This reveals a great deal of useful information: first, there exists a threshold voltage at about $1.2 \mathrm{~V}$, above which the tunneling effect starts to show; second, only within an appropriate range of bias, 


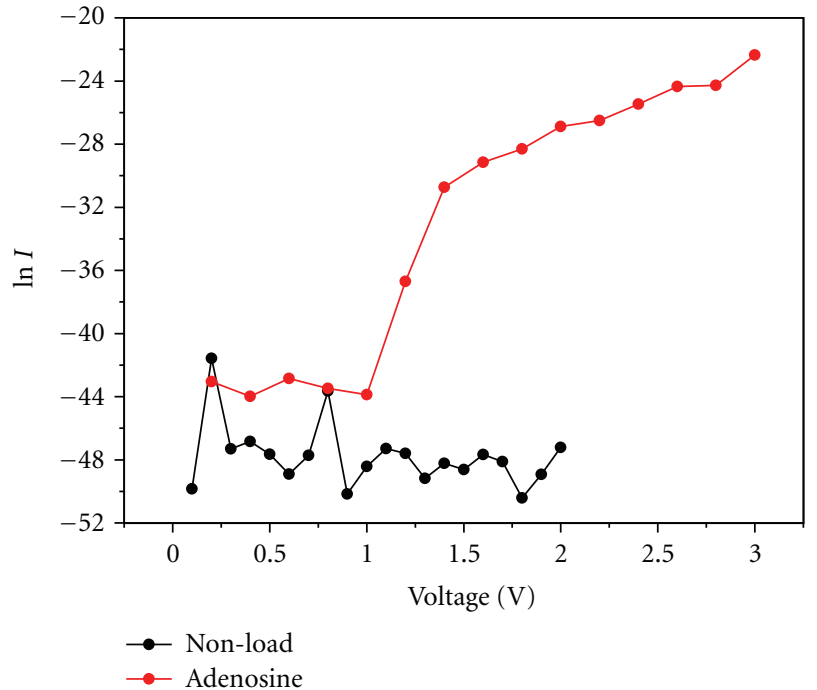

(a)

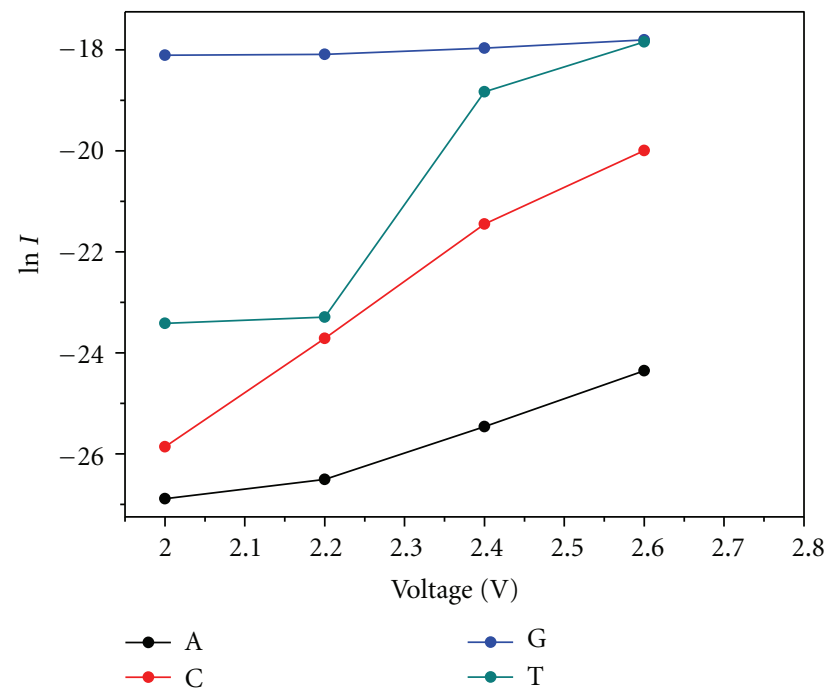

(b)

Figure 3: (a) The $V$ - $\ln I$ curve of adenosine from $0 \mathrm{~V}$ to $3.0 \mathrm{~V}$ with a step of $0.2 \mathrm{~V}$, and the current curve of non-load electrodes system from $0.0 \mathrm{~V}$ to $2.0 \mathrm{~V}$ with a step of $0.1 \mathrm{~V}$. (b) Four distinguishable $V-\ln I$ curves of four nucleotides, A, C, G, T, respectively. All of which express linear relationship from $2.0 \mathrm{~V}$ to $2.6 \mathrm{~V}$ with the same step of $0.2 \mathrm{~V}$. At most voltages, they keep a difference of one or two orders to each other.

which is from $1.4 \mathrm{~V}$ to $3.0 \mathrm{~V}$ in our case, the conclusion before could be held. Our next simulation with four different nucleotides was carried out in this voltage range.

Figure 3(b) represents the transverse current $\ln I-V$ curves of four nucleotides, ranging from $2.0 \mathrm{~V}$ to $2.6 \mathrm{~V}$ with the same step of $0.2 \mathrm{~V}$.

All four curves express linear relationship within this voltage range and show conspicuous distinction between different nucleotides, nearly one or two orders to each other, which agrees with the results by Zwolak and ventra [7], although in a different arrangement of the current intensities. This may result from the different coupling of nucleotides with graphene and nucleotides with $\mathrm{Au}$, which Zwolak used as transverse electrodes in his simulation. The distinct difference in $\ln I$ at the same bias voltage for each nucleotide indicates a potential sequencing approach by using graphene transverse-electrode-based nanopore: we can utilize transverse current to characterize electronic signatures of each nucleotide.

Such transverse current distinction between different nucleotides may result from the geometry dimensions of the different base types. We could see clearly that the current has a same arrangement as the sizes of the four base types (i.e., G, T, C, A). Because the nanopore is just a little bigger than the nucleotides, the tunneling effect is very sensitive to the distance between transverse electrodes. So the small difference in base size will lead to great discrepancy in transverse current. The second reason may be the different interaction between graphene electrodes and base atoms. For instance, the guanosine and cytidine with three hydrogen bonds in the bases have much higher currents than the thymidine and adenosine with two ones. The tunneling current may come from the hydrogen bond on the base, which has been discussed before [24]. Because the thickness of graphene is only single atom, the distance between the electrode and the translocating nucleotide (less than $1 \AA$ ) is much less than that of the neighboring ones (more than $3 \AA$ ), as well as the interaction between them. Thus we need not to consider the interference from the neighboring nucleotides in our calculations.

At last, we could conceive the experimental realization of the proposed method. First, transfer and locate a graphene ribbon, narrow enough to compare with the diameter of the nanopore we expect, on the SiN membrane. Then use transmission electron microscope to fabricate a nanopore on the membrane [25], where the graphene ribbon is located. When the pore is fabricated, the graphene ribbon is cut off into two segments, just on the two sides of the nanopore, and can act as transverse electrodes. After being covered with an insulating layer, the nanopore device with graphene transverse electrodes is fabricated. Here, the most challenge maybe the graphene ribbon is very difficult to manipulate at such narrow scale.

\section{Summary}

In summary, quantum transport simulation using graphene as transverse electrodes was carried out in nanopore-basedDNA analysis, and the obtained $\ln I-V$ curves show exponential relation between the current and the voltage. Moreover, distinguishable distinctions in $\ln I$ under same bias voltage between different nucleotides were demonstrated, indicating the feasibility of the nanopore-based DNA analysis via graphene electrodes. Such findings are fundamentally useful toward the ultimate goal of inexpensive and fast DNA sequencing. 


\section{Acknowledgments}

This project is financially supported by the National Natural Science Foundation of China (Project no. 50902004, 11023003), National 973 Projects (no. 2007CB936202, 2009CB623703, MOST) from China's Ministry of Science and Technology, and the Research Fund for the Doctoral Program of Higher Education.

\section{References}

[1] K. Healy, "Nanopore-based single-molecule DNA analysis," Nanomedicine, vol. 2, no. 4, pp. 459-481, 2007.

[2] J. J. Kasianowicz, E. Brandin, D. Branton, and D. W. Deamer, "Characterization of individual polynucleotide molecules using a membrane channel," Proceedings of the National Academy of Sciences of the United States of America, vol. 93, no. 24, pp. 13770-13773, 1996.

[3] C. Dekker, "Solid-state nanopores," Nature Nanotechnology, vol. 2, no. 4, pp. 209-215, 2007.

[4] K. Healy, B. Schiedt, and I. P. Morrison, "Solid-state nanopore technologies for nanopore-based dna analysis," Nanomedicine, vol. 2, no. 6, pp. 875-897, 2007.

[5] S. Liu, Q. Zhao, Q. Li et al., "Controlled deformation of $\mathrm{Si}_{3} \mathrm{~N}_{4}$ nanopores using focused electron beam in a transmission electron microscope," Nanotechnology, vol. 22, no. 11, Article ID 115302, 2011

[6] D. Branton, D. W. Deamer, A. Marziali et al., "The potential and challenges of nanopore sequencing," Nature Biotechnology, vol. 26, no. 10, pp. 1146-1153, 2008.

[7] M. Zwolak and M. Di Ventra, "Electronic signature of dna nucleotides via transverse transport," Nano Letters, vol. 5, no. 3, pp. 421-424, 2005.

[8] J. Lagerqvist, M. Zwolak, and M. Di Ventra, "Fast dna sequencing via transverse electronic transport," Nano Letters, vol. 6, no. 4, pp. 779-782, 2006.

[9] M. Krems, M. Zwolak, Y. V. Pershin, and M. Di Ventra, "Effect of noise on dna sequencing via transverse electronic transport," Biophysical Journal, vol. 97, no. 7, pp. 1990-1996, 2009.

[10] J. Lagerqvist, M. Zwolak, and M. Di Ventra, "Influence of the environment and probes on rapid DNA sequencing via transverse electronic transport," Biophysical Journal, vol. 93, no. 7, pp. 2384-2390, 2007.

[11] V. Meunier and P. S. Krstić, "Enhancement of the transverse conductance in DNA nucleotides," Journal of Chemical Physics, vol. 128, no. 4, Article ID 041103, 2008.

[12] Y. He, L. Shao, R. H. Scheicher et al., "Differential conductance as a promising approach for rapid DNA sequencing with nanopore-embedded electrodes," Applied Physics Letters, vol. 97, no. 4, Article ID 043701, 2010.

[13] K. S. Novoselov, A. K. Geim, S. V. Morozov et al., "Electric field in atomically thin carbon films," Science, vol. 306, no. 5696, pp. 666-669, 2004.

[14] A. K. Geim, "Graphene: status and prospects," Science, vol. 324, no. 5934, pp. 1530-1534, 2009.

[15] A. K. Geim and K. S. Novoselov, "The rise of graphene," Nature Materials, vol. 6, no. 3, pp. 183-191, 2007.

[16] G. F. Schneider, S. W. Kowalczyk, V. E. Calado et al., "DNA translocation through graphene nanopores," Nano Letters, vol. 10 , no. 8, pp. 3163-3167, 2010.
[17] C. A. Merchant, K. Healy, M. Wanunu et al., "DNA translocation through graphene nanopores," Nano Letters, vol. 10, no. 8, pp. 2915-2921, 2010.

[18] S. Garaj, W. Hubbard, A. Reina, J. Kong, D. Branton, and J. A. Golovchenko, "Graphene as a subnanometre trans-electrode membrane," Nature, vol. 467, no. 7312, pp. 190-193, 2010.

[19] H. W. Postma, "Rapid sequencing of individual DNA molecules in graphene nanogaps," Nano Letters, vol. 10, no. 2, pp. 420-425, 2010.

[20] R. Hoffmann, "An extended Hückel theory. I. Hydrocarbons," The Journal of Chemical Physics, vol. 39, no. 6, pp. 1397-1412, 1963.

[21] M. D. Ventra, Electrical Transport in Nanoscale Systems, Cambridge University Press, Cambridge, UK, 2008.

[22] T. Nelson, B. Zhang, and O. V. Prezhdo, "Detection of nucleic acids with graphene nanopores: $\mathrm{Ab}$ initio characterization of a novel sequencing device," Nano Letters, vol. 10, no. 9, pp. 3237-3242, 2010.

[23] S. Datta, Electrical Transport in Mesoscopic Systems, Cambridge University Press, Cambridge, UK, 1995.

[24] M. H. Lee and O. F. Sankey, "Theory of tunneling across hydrogen-bonded base pairs for DNA recognition and sequencing," Physical Review E, vol. 79, no. 5, Article ID 051911, 10 pages, 2009.

[25] J. Zhang, L. You, H. Ye, and D. Yu, "Fabrication of ultrafine nanostructures with single-nanometre precision in a highresolution transmission electron microscope," Nanotechnology, vol. 18, no. 15, Article ID 155303, 2007. 

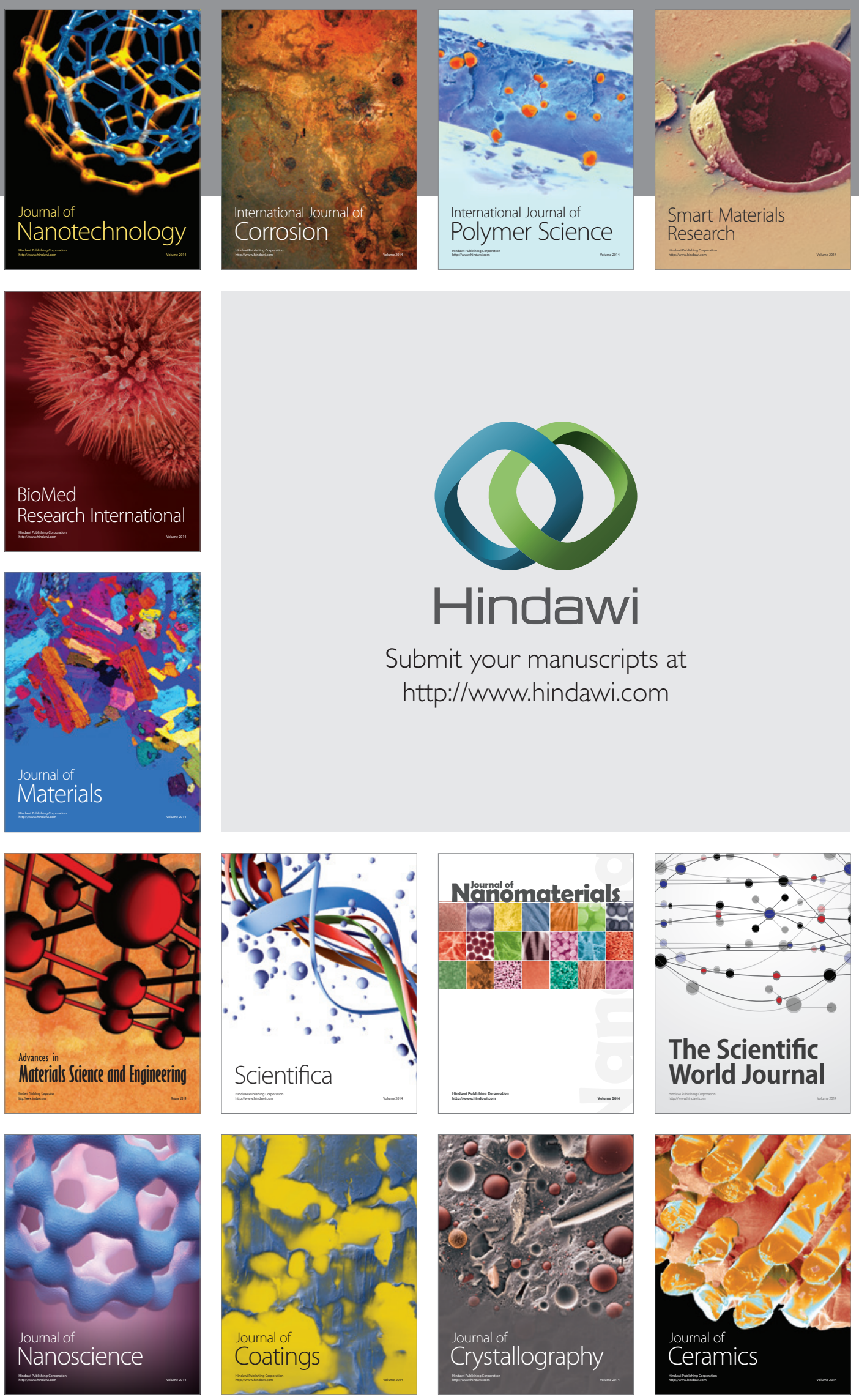

The Scientific World Journal

Submit your manuscripts at

http://www.hindawi.com

\section{World Journal}

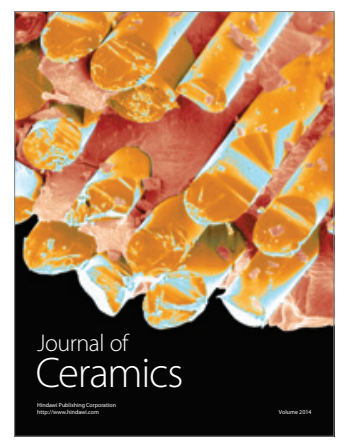

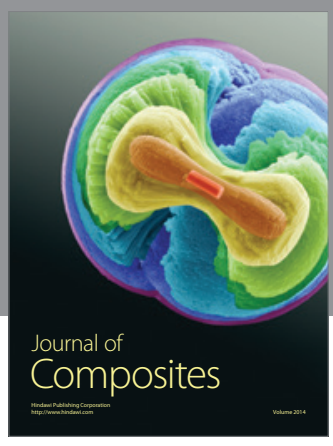
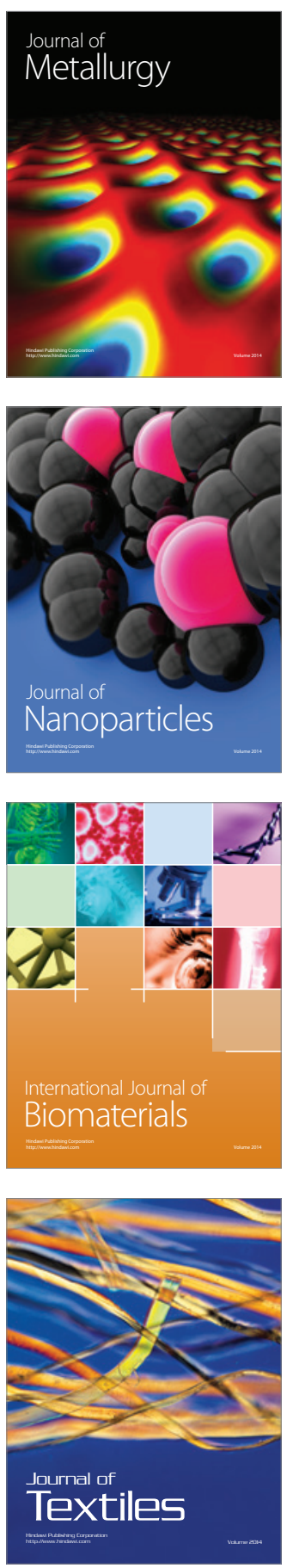